\title{
A unifying convex analysis and switching system approach to consensus with undirected communication graphs
}

\author{
Rafal Goebel*and Ricardo Sanfelice ${ }^{\dagger}$
}

August 6, 2018

\begin{abstract}
Switching between finitely many continuous-time autonomous steepest descent dynamics for convex functions is considered. Convergence of complete solutions to common minimizers of the convex functions, if such minimizers exist, is shown. The convex functions need not be smooth and may be subject to constraints. Since the common minimizers may represent consensus in a multi-agent system modeled by an undirected communication graph, several known results about asymptotic consensus are deduced as special cases. Extensions to time-varying convex functions and to dynamics given by set-valued mappings more general than subdifferentials of convex functions are included.
\end{abstract}

August 6, 2018

\section{Introduction}

This technical note presents a convex analysis and switching systems-based approach to proving convergence to consensus in multi-agent systems or networks modeled by undirected graphs. The approach unifies and generalizes results in, for example, [1, 2], 3], [4], 5], 6], and more, while making weaker assumptions. Even for the linear case, the approach uses no linear or spectral analysis. Certain nonlinearities, given by gradients or subdifferentials or convex functions, convex constraints, and gradient projections fit in the approach.

The convergence result is given for a system that switches between finitely many maximal monotone mappings, which are subdifferentials of convex functions restricted to convex sets. For dynamics given by a single, but multivalued, maximal monotone mapping, for example for the steepest descent for a convex function, existence and uniqueness of solutions, their nonexpansive property, and convergence to equilibria, or minimizers in the steepest descent case, is well studied; see [7] for a classical reference and [8] for a recent survey. The case of a single time-varying maximal monotone mapping has seen less treatment; see [9]. For finitely many convex functions, for continuous-time dynamics driven by

*Department of Mathematics and Statistics, Loyola University Chicago, 1032 W. Sheridan Road, Chicago, IL 60660. Email: rgoebel1@luc.edu. This work was partially supported by the Simons Foundation Grant 315326.

${ }^{\dagger}$ Department of Computer Engineering, University of California, Santa Cruz, CA, 95064, USA. E-mail: ricardo@ucsc.edu. This work was partially supported by the National Science Foundation CAREER Grant ECS-1150306 and by the Air Force Office of Scientific Research YIP Grant FA9550-12-1-0366. 
the minimum norm velocity that can be generated via arbitrary switching between steepest descents, convergence to Pareto-optimal points is expected; see [10] and the references therein. (Common minimizers, if they exist, are Pareto-optimal.) Stability of such points holds [11, for differential inclusions driven by the so-called pseudogradient directions, that include the mentioned minimum norm velocity and are related to descent directions for discrete-time multiobjective optimization [12. These cases, in general, do not apply to switching dynamics. Results on convergence to common minimizers for switching between finitely many convex functions does not appear to have been written down, though similar ideas are, of course, present in alternating projections or alternating proximal point optimization algorithms in discrete-time.

Some convex-analytic methods have been used, to an extent, in the continuous-time consensus setting [3], [5], 6], [13], as well as for the discrete-time case in [14], [15, etc. The setting of this note encompasses, in the undirected graph case, the mentioned continuoustime works while making weaker assumptions. The connection between consensus, gradient flow for a convex function, and convergence of solutions to a minimizer of the function has been made before, in the linear case [2] and beyond [5]. This note carries the idea further. Preliminary work is in [16]. Finally, monotonicity as mentioned above is different from what is considered in monotone systems; see [17] for consensus results in monotone systems.

\section{Main result}

Consider the switching system

$$
\dot{x} \in-M_{q}(x)
$$

where the data is subject to the following assumption, the background for which is presented in Section 3.2 .

Assumption $2.1 Q=\{1,2, \ldots, p\}$, and for every $q \in Q, M_{q}: \mathbb{R}^{n} \rightrightarrows \mathbb{R}^{n}$ is a set-valued mapping given by

$$
M_{q}(x)=\partial f_{q}(x)+N_{C_{q}}(x),
$$

where $f_{q}: \mathbb{R}^{n} \rightarrow \mathbb{R}$ is a convex function; $\partial f_{q}(x)$ is its subdifferential at $x$, in the sense of convex analysis; $C_{q} \subset \mathbb{R}^{n}$ is a nonempty closed convex set; and $N_{C_{q}}(x)$ is the normal cone to $C_{q}$ at $x$.

A switching signal is a function $\sigma:[0, \infty) \rightarrow Q$ such that there exists a sequence $0=t_{0}<t_{1}<t_{2}<\ldots$ such that $\sigma$ is constant on $\left[t_{j}, t_{j+1}\right)$ for $j=0,1,2, \ldots$ Given such a signal $\sigma$, a solution to (11) is a locally absolutely continuous function $\phi: \operatorname{dom} \phi \rightarrow \mathbb{R}^{n}$, where $\operatorname{dom} \phi$ is $[0, T),[0, T]$, or $[0, \infty)$, such that

$$
\dot{\phi}(t) \in M_{\sigma(t)}(\phi(t)) \quad \text { for almost every } t \in \operatorname{dom} \phi .
$$

A solution $\phi$ is maximal if it cannot be extended and complete if dom $\phi=[0, \infty)$. Since $N_{C_{q}}(x)=\emptyset$ if $x \notin C_{q}$, (1) requires $\phi(t) \in C_{\sigma(t)}$ for every $t \in \operatorname{dom} \phi$, maximal solutions $\phi$ may fail to be complete.

Of interest is the behavior of complete solutions to (10) when the functions $f_{q}$ have common minimizers over $C_{q}$. 
Assumption 2.2 The set $A$ is nonempty, where

$$
A:=\bigcap_{q \in Q} A_{q} \quad \text { and } \quad A_{q}:=\underset{x \in C_{q}}{\arg \min } f_{q}(x) .
$$

For every common minimizer $a \in A$, the function $V(x):=\frac{1}{2}\|x-a\|^{2}$ satisfies

$$
\frac{d}{d t} V(\phi(t)) \leq \min _{x \in C_{\sigma(t)}} f_{\sigma(t)}(x)-f_{\sigma(t)}(\phi(t)) \leq 0
$$

along every solution $\phi$ to (11), which follows from the definition of the convex subdifferential (77). In particular, every $a \in A$ is Lyapunov stable for (11). The inequality (2) can be viewed as $\frac{d}{d t} V(\phi(t)) \leq-W_{\sigma(t)}(\phi(t))$, where $W_{q}(x)=f_{q}(x)-\arg \min _{x \in C_{q}} f_{q}(x)$, and it is natural to expect some, or all, $W_{q}$ to asymptotically approach 0 along complete solutions.

Assumption 2.3 The switching signal $\sigma$ is such that, for each $q \in Q$,

$$
\mu\left(T_{q}(\sigma)\right)=\infty \quad \text { where } T_{q}(\sigma):=\{t \in[0, \infty) \mid \sigma(t)=q\} .
$$

Above, $\mu$ is the Lebesgue measure but reduces to the sum of lengths of intervals, since $T_{q}(\sigma)$ is a union of intervals. The assumptions holds, for example, if the switching signal has a positive dwell time $\tau_{D}>0$, i.e., if discontinuities of $\sigma$ are separated by at least $\tau_{D}$, and there exists $T>0$ such that, for every $t \geq 0$, the range of $\sigma$ over $[t, t+T]$ is $Q$. But the assumption is more general.

Under the stated assumptions, the main result is that every complete solution to (1) converges to a common minimizer in $A$.

Theorem 2.4 Under assumptions 2.1, 2.2, and 2.3 every complete solution to (1) is such that $\lim _{t \rightarrow \infty} x(t)$ exists and belongs to $A$.

In the setting of Theorem 2.4, since every $a \in A$ is Lyapunov stable, the set $A$ has the property called pointwise asymptotic stability, also known as semistability. See [18] and the references therein.

Of particular interest, motivated by consensus questions, is when and how the set of common minimizers $A$ is related to the consensus subspace. Let $n=k m$, where $k$ represents the number of $m$-dimensional agents. For convenience, $x \in \mathbb{R}^{n}$ is $\left(x_{1}, x_{2}, \ldots, x_{k}\right)$, with $x_{i} \in \mathbb{R}^{m}$, and the consensus subspace is

$$
C S:=\left\{x \in \mathbb{R}^{n} \mid x_{1}=x_{2}=\cdots=x_{k}\right\}
$$

If complete solutions to (11) are such that their limits exist and are in $C S$, it is said that the agents reach consensus.

Example 2.5 Let $f: \mathbb{R}^{n} \rightarrow \mathbb{R}$ be

$$
f(x)=\frac{1}{4} \sum_{i, j=1}^{k} a_{i j}\left\|x_{i}-x_{j}\right\|_{p}^{p},
$$

where, for $i, j=1, \ldots, k, a_{i j}=a_{j i} \geq 0, p \in[1, \infty)$, and $\|\cdot\|_{p}$ is the usual $p$-norm. Let $C=\mathbb{R}^{n}$. Then $f$ is a convex function. For $p>1$, $\partial f$ reduces to $\nabla f, N_{C}(x)=\{0\}$ for all 
$x \in \mathbb{R}^{n}, M$ reduces to $\nabla f$, and (10) becomes $\dot{x}=-\nabla f(x)$. For $p=2$, more explicitly, this differential equation is, for $i=1, \ldots, k$,

$$
\dot{x}_{i}=\frac{1}{2} \sum_{j=1}^{k} a_{i j}\left(x_{j}-x_{i}\right) .
$$

For $p=1$, (10) turns to

$$
\dot{x}_{i}=\sum_{j=1}^{k} a_{i j} \operatorname{sign}\left(x_{j}-x_{i}\right), \quad i=1, \ldots, k,
$$

where sign is taken coordinate-wise and, by convention, $\operatorname{sign}(0)=0$. (The convex subdifferential of the absolute value is set valued at 0 , and equals $[-1,1]$, but this does not affect the solutions.) Second-order controllers with the sign function are, for example, in [19].

Here, $A=\arg \min f$, and clearly $C S \subset A$. If the communication graph between agents is connected, and $a_{i j}=a_{j i}>0$ for every undirected edge between $i$-th and $j$-th agent, then $A \subset C S$ and thus $A=C S$. Indeed, in such a case, for any two agents, say $i$-th and $j$-th, there exist agents $i=i_{0}, i_{1}, i_{2}, \ldots, i_{L}=j$ such that there is an edge between $i_{l-1}$ and $i_{l}$, and so $a_{i_{l-1}, i_{l}}=a_{i_{l}, i_{l-1}}>0$, for each $l=1,2, \ldots, L ; f(x)=\min f=0$ is possible only if $a_{i-1, i}\left(x_{i-1}-x_{i}\right)^{2}=0$ for each $l=1,2, \ldots, L$, and so $x_{i-1}=x_{i}$ for each $l=1,2, \ldots, L$, and consequently, $x_{i}=x_{j}$. Theorem 2.4 implies that, in this case, agents reach consensus. This conclusion is, of course, well-known; see, for example, [2, Lemma 1].

On the other hand, if $C S \neq A$, which requires that the communication graph be not connected, for some initial conditions (in particular, those in $A \backslash C S$, which are equilibria of (3) ), the agents do not reach consensus.

Example 2.6 Let $f: \mathbb{R}^{n} \rightarrow \mathbb{R}$ be

$$
f(x)=\frac{1}{4} \sum_{i, j=1}^{k} a_{i j}\left\|x_{i}-x_{j}\right\|_{\infty}^{2},
$$

where, for $i, j=1, \ldots, k, a_{i j}=a_{j i} \geq 0$, and $\|u\|_{\infty}=\max _{i=1,2, \ldots, m}\left|u_{i}\right|$. Let $C=\mathbb{R}^{n}$. Then $f$ is a convex function and $\dot{x} \in-\partial f(x)$ becomes

$$
\dot{x}_{i}=\sum_{j=1}^{k} a_{i j}\left(x_{j, l(i, j)}-x_{i, l(i, j)}\right),
$$

when $x$ is such that, for every $i, j=1,2, \ldots, k$, there exists a unique $l(i, j) \in\{1,2, \ldots, m\}$ such that $\left\|x_{i}-x_{j}\right\|_{\infty}=\left|x_{i, l(i, j)}-x_{j, l(i, j)}\right|$. Then, the contribution of the $j$-th agent to the velocity of the $i$-th one is only in the coordinate in which their positions differ the most. At other points $x, f$ is not differentiable and $\dot{x}$ can be found as the minimum norm element of $-\partial f(x)$.

If the norm is not squared, in the formula for $f$, then the dynamics, at $x$ as specified above, is

$$
\dot{x}_{i}=\frac{1}{2} \sum_{j=1}^{k} a_{i j} \operatorname{sign}\left(x_{j, l(i, j)}-x_{i, l(i, j)}\right) .
$$


Example 2.7 Let $f_{q}: \mathbb{R}^{n} \rightarrow \mathbb{R}$ be given by

$$
f_{q}(x)=\frac{1}{4} \sum_{i, j=1}^{k} a_{i j}(q)\left(x_{i}-x_{j}\right)^{2},
$$

where, for $i, j=1, \ldots, k, a_{i j}(q)=a_{j i}(q) \geq 0$. Let $C_{q}=\mathbb{R}^{n}$. Then, as suggested by Example 2.5, the switching system (10) is

$$
\dot{x}_{i}=\sum_{j=1}^{k} a_{i j}(q)\left(x_{j}-x_{i}\right) .
$$

Here, $A=\cap_{q \in Q} \arg \min f_{q}$. Clearly, $C S \subset A_{q}$ for each $q$ and thus $C S \subset A$. If

(CG) the graph given by the union of the communication graphs associated to each $q \in Q$ is connected, and, for each $q \in Q, a_{i j}(q)=a_{j i}(q)>0$ for every undirected edge between $i$-th and $j$-th agent in the $q$-th graph,

then $A \subset C S$ and thus $A=C S$. Under Assumption 2.3, Theorem 2.4 implies that, in this case, agents reach consensus.

Example 2.8 Let $f_{q}: \mathbb{R}^{n} \rightarrow \mathbb{R}$ be

$$
f_{q}(x)=\sum_{i=1}^{k} g_{i}\left(x_{i}\right)+\frac{1}{4} \sum_{i, j=1}^{k} a_{i j}(q)\left(x_{i}-x_{j}\right)^{2},
$$

where $g_{i}: \mathbb{R}^{n} \rightarrow \mathbb{R}$ are differentiable convex functions and $a_{i j}(q)=a_{j i}(q) \geq 0$. Let $C_{q}=\mathbb{R}^{n}$. Then, the switching system (1) is

$$
\dot{x}_{i}=-\nabla g_{i}\left(x_{i}\right)+\sum_{j=1}^{k} a_{i j}(q)\left(x_{j}-x_{i}\right),
$$

and $-\nabla g_{i}\left(x_{i}\right)$ can be considered as local dynamics of the $i$-th agent. If $D:=\cap_{i=1}^{k} D_{i} \neq \emptyset$, where $D_{i}:=\arg \min g_{i}$, then for every $\xi \in D,(\xi, \xi, \ldots, \xi) \in A$. If, additionally, (CG) holds, then every $a \in A$ has the form $(\xi, \xi, \ldots, \xi)$ with $\xi \in D$. Thus, under both conditions, agents reach consensus while also finding a common minimizer to $g_{i}$. This recovers $[\underline{5}$, Theorem 4.1]; in fact generalizes it to the switching setting.

Furthermore, if $g_{i}\left(x_{i}\right)=\frac{1}{2} \operatorname{dist}_{D_{i}}\left(x_{i}\right)^{2}$, where $D_{i} \subset \mathbb{R}^{m}$ is a nonempty closed and convex set, and $\operatorname{dist}_{D_{i}}\left(x_{i}\right)$ is the distance of $x_{i}$ from $D_{i}$, then $\nabla g_{i}\left(x_{i}\right)=x_{i}-P_{D_{i}}\left(x_{i}\right)$, where $P_{D_{i}}\left(x_{i}\right)$ is the projection of $x_{i}$ onto $D_{i}$, i.e., the point in $D_{i}$ closest to $x_{i}$, and (5) becomes

$$
\dot{x}_{i}=P_{D_{i}}\left(x_{i}\right)-x_{i}+\sum_{j=1}^{k} a_{i j}(q)\left(x_{j}-x_{i}\right) .
$$

If $D:=\cap_{i=1}^{k} D_{i} \neq \emptyset$ and (CG) holds, every $a \in A$ has the form $(\xi, \xi, \ldots, \xi)$ with $\xi \in D$, and agents reach consensus while finding a point in this intersection. This is an autonomous version of a result in [3]; the time-varying case can be addressed as in Section 4.1 . 
Example 2.9 Let $f_{q}$ be as in Example 2.7 and $C$ be given by

$$
C=C_{1} \times C_{2} \times \cdots \times C_{k}
$$

for nonempty closed convex $C_{i} \subset \mathbb{R}^{m}$. Then, the switching system (1) has the same solutions as the system

$$
\dot{x}_{i}=P_{T_{C_{i}}\left(x_{i}\right)}\left(\sum_{j=1}^{k} a_{i j}(q)\left(x_{j}-x_{i}\right)\right),
$$

where $P_{T_{C_{i}}\left(x_{i}\right)}(v)$ is the projection of $v$ onto the space tangent to $C_{i}$ at $x_{i}$; see Section 3.2 for justification. Thus, (11) reduces to (4) projected onto $C_{i}$. Note that the projection can be done locally, i.e., by each agent independently. This is the continuous-time version of the dynamics considered, for example, in [14].

\section{Proof of the main result}

\subsection{Auxiliary convergence result}

Theorem 3.1 Let $Q=\{1,2, \ldots, p\}$. Let $\sigma$ be a switching signal that satisfies Assumption 2.3 and $\phi:[0, \infty) \rightarrow \mathbb{R}^{n}$ be a bounded, uniformly continuous, and locally absolutely continuous function. Suppose that there exist

- a differentiable function $V: \mathbb{R}^{n} \rightarrow \mathbb{R}$ which is bounded below;

- for every $q \in Q$, a lower semicontinuous function $W_{q}: \mathbb{R}^{n} \rightarrow[0, \infty)$;

such that,

(a) for almost all $t \in[0, \infty)$,

$$
\frac{d}{d t} V(\phi(t)) \leq-W_{\sigma(t)}(\phi(t))
$$

(b) $A:=\bigcap_{q \in Q} A_{q} \neq \emptyset$ where $A_{q}:=\arg \min W_{q}$;

(c) for every $q \in Q$, on every interval on which $\sigma(t)=q$, for every $a_{q} \in A_{q}, t \mapsto$ $\left\|\phi(t)-a_{q}\right\|$ is nonincreasing.

Then $\phi(t)$ converges, as $t \rightarrow \infty$ to a point in $A$.

The result above borrows some ideas from results involving multiple Lyapunov functions, for example [20, Theorem 7], and possibly other related results [21], but $V$ and $W_{q}$ are not related through observability or detectability-like conditions, and assumption (c) is quite specific for the subdifferential flow for a convex function and unusual for the multiple Lyapunov functions results. Without this assumption, the result fails. Indeed, consider $\dot{x}=y, \dot{y}=-x, V(x, y)=x^{2}+y^{2}$, and $W_{q}$, for $q=1,2,3,4$ being the distance from the $q$-th quadrant. For any complete (and periodic!) solution $\phi$ not from the origin, with $\sigma(t)=q$ if $\phi(t)$ is in the $q$-th quadrant, all assumptions except (d) are satisfied, in 
particular (6) holds with both sides equal to 0 . Note that also, when $\sigma(t)=q$, then the distance of $\phi(t)$ from the $q$-th quadrant is 0 and thus nondecreasing. But the conclusion fails: $A$ is the origin.

Proof. Let the sets $T_{q}(\sigma)$ come from Assumption 2.3. For each $q \in Q$, there exists a sequence $t_{q, j} \rightarrow \infty$ as $j \rightarrow \infty$ such that $t_{q, j} \in T_{q}(\sigma)$ and $W_{q}\left(\phi\left(t_{q, j}\right)\right) \rightarrow 0$. Indeed, in the opposite case there exists $\delta>0$ such that, for all large enough $t \in T_{q}(\sigma), W_{q}(\phi(t)) \geq \delta$ and thus $\frac{d}{d t} V(\phi(t)) \leq-\delta$. Since $\mu\left(T_{q}(\sigma)\right)=\infty$, this contradicts $V$ being bounded below. Since $\phi$ is bounded, without loss of generality one can assume that $\phi\left(t_{q, j}\right)$ converge, as $j \rightarrow \infty$, to a limit denoted $a_{q}$. By lower semicontinuity and nonnegativity of $W_{q}, W\left(a_{q}\right)=0$ and $a_{q} \in A_{q}$.

Let $Q_{1}=\left\{q \in Q \mid a_{1} \in A_{q}\right\}$. It will be shown that $Q_{1}=Q$. Suppose that $Q_{1} \neq Q$ and, without loss of generality, that $2 \notin Q_{1}$. Let $d>0$ be such that

$$
d<\min _{q \notin Q_{1}} \min _{x \in A_{q}}\left\|x-a_{1}\right\|
$$

and define

$$
\delta=\min _{q \notin Q_{1}} \min _{x:\left\|x-a_{1}\right\| \leq d} W_{q}(x),
$$

which is positive since $W_{q}$ are continuous and if $\left\|x-a_{1}\right\| \leq d$ then $x \notin A_{q}$ for $q \notin Q_{1}$. Recall that $\phi\left(t_{1, j}\right) \rightarrow a_{1}$ and $\phi\left(t_{2, j}\right) \rightarrow a_{2}$ as $j \rightarrow \infty$. Thus, there exist sequences $\tau_{j}, \tau_{j}^{\prime}$ with $\tau_{j}<\tau_{j}^{\prime}<\tau_{j+1}$ such that, for each $j=1,2, \ldots$,

$$
\begin{gathered}
\left\|\phi\left(\tau_{j}\right)-a_{1}\right\|=d / 2, \quad\left\|\phi\left(\tau_{j}^{\prime}\right)-a_{1}\right\|=d, \\
d / 2 \leq\left\|\phi(t)-a_{1}\right\| \leq d \forall t \in\left[\tau_{j}, \tau_{j}^{\prime}\right] .
\end{gathered}
$$

For almost all $t \in\left[\tau_{j}, \tau_{j}^{\prime}\right]$ such that $\sigma(t) \in Q_{1}$, i.e., such that $a_{1} \in A_{\sigma(t)},\left\|\phi(t)-a_{1}\right\|$ is nonincreasing. Let $S_{j}:=\left\{t \in\left[\tau_{j}, \tau_{j}^{\prime}\right] \mid \sigma(t) \notin Q_{1}\right\}$. Since $\left\|\phi\left(\tau_{j}^{\prime}\right)-\phi\left(\tau_{j}\right)\right\| \geq d / 2$ and $\phi$ is uniformly continuous, there exists $\eta>0$ such that, for each $j, \mu\left(S_{j}\right) \geq \eta$. But for almost all $t \in S:=\bigcup_{j=1}^{\infty} S_{j}, \frac{d}{d t} V(\phi(t)) \leq-\delta$. Since $\mu(S)=\infty$, this is a contradiction! Consequently, $Q_{1}=Q$ and $a_{1} \in A$. Assumption (c) ensures now that $\left\|\phi(t)-a_{1}\right\|$ is nonincreasing and hence $\phi(t)$ converges to $a_{1}$.

\subsection{Convex analysis background}

For details on the convex analysis material collected below, see [22]. For details on differential inclusions, including (8), see [23], [24].

Let $g: \mathbb{R}^{n} \rightarrow \mathbb{R} \cup\{\infty\}$ be a proper (i.e., finite somewhere), lower semicontinuous (lsc), and convex function. Let dom $g$ be the effective domain of $g$, i.e. the set $\left\{x \in \mathbb{R}^{n} \mid g(x)<\right.$ $\infty\}$. The convex subdifferential mapping of $g$ is the set-valued mapping $\partial g: \mathbb{R}^{n} \rightrightarrows \mathbb{R}^{n}$, with $\partial g(x)$ given by

$$
\left\{y \in \mathbb{R}^{n} \mid g\left(x^{\prime}\right) \geq g(x)+y \cdot\left(x^{\prime}-x\right) \forall x^{\prime} \in \mathbb{R}^{n}\right\} .
$$

The subdifferential mapping $\partial g$ is maximal monotone, and consequently, for the differential inclusion

$$
\dot{x} \in-\partial g(x)
$$

one has 
(a) For every $x_{0}$ in the closure of dom $g$ there exists a unique maximal solution to (8) with $\phi(0)=x_{0}$ and this solution is complete.

(b) For any two complete solutions $\phi, \psi$ to (8), $t \mapsto\|\phi(t)-\psi(t)\|$ is nonincreasing. In particular, the solutions to (8) depend continuously on initial conditions, in the uniform norm over $[0, \infty)$.

(c) For every solution $\phi$ to (맘 and for almost all $t \geq 0$

$$
\dot{x}(t)=m(-\partial g(x(t)))
$$

where $m(S)$ is the element of the closed set $S$ with minimum norm.

Now let $g: \mathbb{R}^{n} \rightarrow \mathbb{R}$ be convex and let $D \subset \mathbb{R}$ be a nonempty, closed, and convex set. For such $g, \partial g$ is locally bounded. Define $g_{D}: \mathbb{R}^{n} \rightarrow \mathbb{R} \cup\{\infty\}$ by

$$
g^{D}(x)=\left\{\begin{array}{ccc}
g(x) & \text { if } & x \in D \\
\infty & \text { if } & x \notin D
\end{array}\right.
$$

Then $g^{D}$ is proper, lower semicontinuous, and convex, and its subdifferential is given by

$$
\partial g^{D}(x)=\left\{\begin{array}{ccc}
\partial g(x) & \text { if } & x \in \operatorname{int} D \\
\partial g(x)+N_{D}(x) & \text { if } & x \in \operatorname{bdry} D \\
\emptyset & \text { if } & x \notin D
\end{array}\right.
$$

Above, int $D$ and bdry $D$ stand for the interior and the boundary of $D$, and $N_{D}(x)$ is the normal cone to $D$ at $x$, given by

$$
N_{D}(x)=\left\{v \in \mathbb{R}^{n} \mid v \cdot\left(x^{\prime}-x\right) \leq 0 \forall x^{\prime} \in D\right\} .
$$

Since $0 \in N_{D}(x)$ for every $x \in D$, the minimum norm element of $-\partial g(x)-N_{D}(x)$ is locally bounded. The general facts above can be applied to $M_{q}=\partial f_{q}+N_{C_{q}}$.

Proposition 3.2 Under Assumption 2.1, given a switching signal $\sigma:[0, \infty) \rightarrow Q$, and $x_{0} \in C_{\sigma(0)}$, there exists a unique maximal solution $\phi: \operatorname{dom} \phi \rightarrow \mathbb{R}^{n}$ to (1) with $\phi(0)=x_{0}$. If this solution is bounded, it is uniformly continuous. This solution is complete if, for example, $C_{1}=C_{2}=\cdots=C_{p}$.

If $g: \mathbb{R}^{n} \rightarrow \mathbb{R}$ is differentiable, then for $x \in \operatorname{bdry} D$, the minimum norm element of $-\nabla g(x)-N_{D}(x)$ is the same as the projection of $-\nabla g(x)$ onto the tangent cone to $D$ at $x$ :

$$
-m\left(\nabla g(x)+N_{D}(x)\right)=P_{T_{D}(x)}(-\nabla g(x)),
$$

see [25] or the recent [26, Corollary 2]. Thus, solutions to $\dot{x} \in-\partial g^{D}(x)$ are the same as solutions to projected gradient dynamics $\dot{x}=P_{T_{D}(x)}(-\nabla g(x))$. Above, the tangent cone to $D$ at $x$ is

$$
T_{D}(x)=\left\{u \in \mathbb{R}^{n} \mid u \cdot v \leq 0 \forall v \in N_{D}(x)\right\}
$$




\subsection{Proof of Theorem 2.4}

Take $a \in A$. Let $V(x)=\frac{1}{2}\|x-a\|^{2}$. Let $\sigma$ be a switching signal and $\phi: \operatorname{dom} \phi \rightarrow \mathbb{R}^{n}$ a solution to (1). Then, since $M_{q}=\partial f_{q}^{C_{q}}$ where $f_{q}^{C_{q}}$ is constructed from $f_{q}$ and $C_{q}$ via (9),

$$
\frac{d}{d t} V(\phi(t))=(\phi(t)-a) \cdot \dot{\phi}(t) \leq f_{\sigma(t)}^{C_{\sigma(t)}}(a)-f_{\sigma(t)}^{C_{\sigma(t)}}(\phi(t)) \leq 0
$$

where the first inequality above comes directly from the definition of the subdifferential (17). This confirms Lyapunov stability of $a$ for (11) and ensures that $\phi$ is bounded. Let $\phi$ be complete. For $q \in Q$, let $W_{q}: \mathbb{R}^{n} \rightarrow[0, \infty)$ be given by $W_{q}(x)=f_{q}^{C_{q}}(x)-\min _{x \in C_{q}} f_{q}(x)$. Note that $W_{q}(x)=f_{q}(x)-\min _{x \in C_{q}} f_{q}(x)$ for $x \in C_{q}, W_{q}(x)=\infty$ otherwise, and so $W_{q}$ is lsc. Then $\phi$ satisfies (6) and Theorem 3.1 implies the result.

\section{Extensions}

Without Assumption 2.3, one can still conclude convergence of solutions to (11), with limits in a set that depends on the switching signal. Given a switching signal $\sigma$, let

$$
Q_{\infty}(\sigma):=\left\{q \in Q \mid T_{q}(\sigma)=\infty\right\}, \quad A_{\infty}(\sigma):=\bigcap_{q \in Q_{\infty}} A_{q} .
$$

Corollary 4.1 Under assumptions 2.1 and 2.2, given a switching signal $\sigma$, every complete solution to (1) is such that $\lim _{t \rightarrow \infty} x(t)$ exists and belongs to $A_{\infty}(\sigma)$.

This can be deduced from the proof of Theorem 2.4. For consensus purposes, the question then becomes whether $A_{\infty} \subset C S$.

Two other extensions of Theorem 2.4 conclude this technical note.

\subsection{Switching and mildly time-varying case}

Consider the switching system

$$
\dot{x} \in-M_{q}(x, t) \text {. }
$$

Assumption 4.2 $Q=\{1,2, \ldots, p\}$, and for every $q \in Q, M_{q}: \mathbb{R}^{n} \times[0, \infty) \rightrightarrows \mathbb{R}^{n}$ is a set-valued mapping given by

$$
M_{q}(x, t)=\partial f_{q}(x, t)+N_{C_{q}}(x)
$$

where $f_{q}: \mathbb{R}^{n} \times[0, \infty) \rightarrow \mathbb{R}$ is a function convex in $x$ for each fixed $t ; \partial f_{q}(x, t)$ is the subdifferential, in the sense of convex analysis, of $x \mapsto f_{q}(x, t)$ at $x ; C_{q} \subset \mathbb{R}$ is a nonempty closed convex set; and $N_{C_{q}}(x)$ is the normal cone to $C_{q}$ at $x$. Additionally

(a) for each $q \in Q$, there exists a closed convex set $A_{q} \subset \mathbb{R}^{n}$ such that, for every $t \in[0, \infty)$,

$$
\underset{x \in C_{q}}{\arg \min } f_{q}(x, t)=A_{q}
$$

(b) the set $A:=\bigcap_{q \in Q} A_{q}$ is nonempty;

(c) for each $q \in Q$, there exists a convex function $g_{q}: \mathbb{R}^{n} \rightarrow \mathbb{R}$ such that 


$$
\begin{aligned}
& \text { - } \arg \min _{x \in C_{q}} g_{q}(x)=A_{q} ; \\
& \text { - for every } x \in \mathbb{R}^{n}, t \in[0, \infty), g_{q}(x) \leq f_{q}(x, t) ;
\end{aligned}
$$

(d) for each $q \in Q, \partial f_{q}(x, t)$ are locally bounded in $x$ uniformly in $t \in[0, \infty)$.

Theorem 4.3 Under assumptions 4.2 and 2.3, every complete solution to (10) is such that $\lim _{t \rightarrow \infty} x(t)$ exists and belongs to $A$.

Proof. The proof relies on Theorem 3.1 and is identical to that of Theorem 2.4, after noting that the same $V$ leads to

$$
\begin{aligned}
\frac{d}{d t} V(\phi(t)) & \leq f_{\sigma(t)}^{C_{\sigma(t)}}(a, t)-f_{\sigma(t)}^{C_{\sigma(t)}}(\phi(t), t) \\
& \leq g_{\sigma(t)}^{C_{\sigma(t)}}(a)-g_{\sigma(t)}^{C_{\sigma(t)}}(\phi(t)) \leq 0
\end{aligned}
$$

one can take $W_{q}: \mathbb{R}^{n} \rightarrow[0, \infty)$ given by $W_{q}(x)=g_{q}^{C_{q}}(x)-\min _{x \in C_{q}} g_{q}(x)$, and uniform continuity of every bounded solution comes from Assumption 4.2 (d).

Example 4.4 Let $f_{q}: \mathbb{R}^{n} \times[0, \infty) \rightarrow \mathbb{R}$ be given by

$$
f_{q}(x, t)=\frac{1}{4} \sum_{i, j=1}^{k} a_{i j}(q, t)\left(x_{i}-x_{j}\right)^{2},
$$

where, for $i, j=1, \ldots, k, a_{i j}(q)=a_{j i}(q) \geq 0$. Let $C_{q}=\mathbb{R}^{n}$. Then (10) is, for $i=1, \ldots, k$,

$$
\dot{x}_{i}=\sum_{j=1}^{k} a_{i j}(q, t)\left(x_{j}-x_{i}\right) .
$$

Suppose that there exist $a_{*}, a^{*}>0$ such that, for every $q$, every $i, j=1,2, \ldots, k$, either $a_{i j}(q, t)=0$ for all $t \geq 0$ or

$$
a_{*} \leq a_{i j}(q, t) \leq a^{*} \text { for all } t \geq 0 .
$$

Assumption like the above is made, for example, by [3], [5], 6]. Then $g_{q}: \mathbb{R}^{n} \rightarrow \mathbb{R}$ fitting Assumption 4.2 (c) is

$$
g_{q}(x)=\frac{1}{4} \sum_{i, j=1}^{k} a_{*}\left(x_{i}-x_{j}\right)^{2} .
$$

Bounds on $a_{i j}(t)$ ensure that Assumption 4.2 (d) holds.

As in the autonomous case, $C S \subset A_{q}$ for each $q$ and thus $C S \subset A$. If the union of $q$-th communication graphs between agents, over all $q \in Q$, is connected, and and $a_{i j}(q, t)=a_{j i}(q, t)>0$ for all $t \in[0, \infty)$ for every undirected edge between $i$-th and $j$-th agent in the $q$-th graph, then $A \subset C S$ and thus $A=C S$. Under Assumption 2.3, Theorem 4.3 implies that, in this case, agents reach consensus.

Extending the arguments above to include local dynamics as in Example 2.8 recovers [5. Theorem 5.3]. Considering local dynamics given by $\dot{x}_{i}=P_{D_{i}}\left(x_{i}\right)-x_{i}$, as at the end of Example 2.8, recovers [3, Theorem 3.1]. 


\subsection{Demipositive maximal monotone dynamics}

Demipositivity generalizes the property of the subdifferential of a convex function $f$ that guarantees that solutions to $\dot{x} \in-\partial f$ converge to minima of $f$. Original definition given by [27. was in an infinite-dimensional Hilbert space setting. Simplified to the finitedimensional case, the definition is this: a maximal monotone mapping $M: \mathbb{R}^{n} \rightrightarrows \mathbb{R}^{n}$ is demipositive if there exists $a \in M^{-1}(0)$ such that, if $v \cdot(x-a)=0$ for some $v \in M(x)$ then $x \in M^{-1}(0)$. For a proper, lsc, and convex $f, \partial f$ is demipositive. For a differentiable function $h(x, y)$ strictly convex in $x$ and strictly concave in $y$, the mapping $(x, y) \mapsto$ $\left(\nabla_{x} h(x, y),-\nabla_{y} h(x, y)\right)$ is demipositive, but without strictness, this may fail: that same mapping for $h(x, y)=x^{2}+x y$ is not demipositive.

Let $M: \mathbb{R}^{n} \rightrightarrows \mathbb{R}^{n}$ be a demipositive maximal monotone mapping with $M^{-1}(0)$ compact. Let $K \subset \mathbb{R}^{n}$ be compact and such that $K \cap M^{-1}(0)=\emptyset$. Then

$$
\inf _{a \in M^{-1}(0)} \inf _{x \in K} \inf _{v \in M(x)} v \cdot(x-a)
$$

is positive and thus there exists a lsc function $W: \mathbb{R}^{n} \rightarrow[0, \infty)$ with $W(x)=0$ if and only if $x \in M^{-1}(0)$ and such that $\frac{d}{d t} \frac{1}{2}\|\phi(t)-a\|^{2} \leq-W(\phi(t))$ for every solution to $\dot{x} \in-M(x)$, every $a \in M^{-1}(0)$. Theorem 3.1 can be then used to prove:

Theorem 4.5 Let $Q=1,2, \ldots, p$. For each $q \in Q$, let $M_{q}: \mathbb{R}^{n} \rightrightarrows \mathbb{R}^{n}$ be a demipositive maximal monotone mapping. Suppose that

$$
A:=\bigcap_{q \in Q} M^{-1}(0) \neq \emptyset
$$

Let $\sigma$ be a switching signal that satisfies Assumption 2.3 and $\phi:[0, \infty) \rightarrow \mathbb{R}^{n}$ be a uniformly continuous solution to (1). Then $\phi(t)$ converges, as $t \rightarrow \infty$ to a point in $A$.

\section{Acknowledgment}

The work by R. Goebel was partially supported by the Simons Foundation Grant 315326 . This work by R. Sanfelice was partially supported by the National Science Foundation under CAREER Grant no. ECS-1450484, Grant no. ECS-1710621, and Grant no. CNS1544396, by the Air Force Office of Scientific Research under Grant no. FA9550-16-1-0015, and by the Air Force Research Laboratory under Grant no. FA9453-16-1-0053.

\section{References}

[1] K.-K. Oh, M.-C. Park, and H.-S. Ahn. A survey of multi-agent formation control. Automatica J. IFAC, 53:424-440, 2015.

[2] R. Olfati-Saber, J.A. Fax, and R.M. Murray. Consensus and cooperation in networked multi-agent systems. Proceedings of the IEEE, 95(1):215-233, 2007.

[3] G. Shi, K.H. Johansson, and Y. Hong. Reaching an optimal consensus: dynamical systems that compute intersections of convex sets. IEEE Trans. Automat. Control, 58(3):610-622, 2013. 
[4] Z. Qiu, S. Liu, and L. Xie. Distributed constrained optimal consensus of multi-agent systems. Automatica J. IFAC, 68:209-215, 2016.

[5] G. Shi, A. Proutiere, and K.H. Johansson. Network synchronization with convexity. SIAM J. Control Optim., 53(6):3562-3583, 2015.

[6] T. Yang, Z. Meng, G. Shi, Y. Hong, and K.H. Johansson. Network synchronization with nonlinear dynamics and switching interactions. IEEE Trans. Automat. Control, 61(10):3103-3108, 2016.

[7] H. Brézis. Opérateurs maximaux monotones et semi-groupes de contractions dans les espaces de Hilbert. North-Holland Publishing Co., Amsterdam-London; American Elsevier Publishing Co., Inc., New York, 1973.

[8] J. Peypouquet and S. Sorin. Evolution equations for maximal monotone operators: asymptotic analysis in continuous and discrete time. J. Convex Anal., 17(3-4):1113$1163,2010$.

[9] H. Attouch, A. Cabot, and M.-O. Czarnecki. Asymptotic behavior of nonautonomous monotone and subgradient evolution equations. Trans. Amer. Math. Soc., 370(2):755790, 2018.

[10] H. Attouch, G. Garrigos, and X. Goudou. A dynamic gradient approach to Pareto optimization with nonsmooth convex objective functions. J. Math. Anal. Appl., 422(1):741-771, 2015.

[11] E. Miglierina. Slow solutions of a differential inclusion and vector optimization. SetValued Anal., 12(3):345-356, 2004.

[12] J. Fliege and B.F. Svaiter. Steepest descent methods for multicriteria optimization. Math. Methods Oper. Res., 51(3):479-494, 2000.

[13] X. Zeng, P. Yi, and Y. Hong. Distributed continuous-time algorithm for constrained convex optimizations via nonsmooth analysis approach. IEEE Trans. Automat. Control, 62(10):5227-5233, 2017.

[14] A. Nedić, A. Ozdaglar, and P.A. Parrilo. Constrained consensus and optimization in multi-agent networks. IEEE Trans. Automat. Control, 55(4):922-938, 2010.

[15] M. Zhu and S. Martínez. On distributed convex optimization under inequality and equality constraints. IEEE Trans. Automat. Control, 57(1):151-164, 2012.

[16] R. Goebel and R.G. Sanfelice. Applications of convex analysis to consensus algorithms, pointwise asymptotic stability, and its robustness. In Proc. 57th IEEE Conference on Decision and Control, 2018. submitted, available at https://hybrid.soe.ucsc.edu/files/preprints/GS-CDC18.pdf, password PASgradient.

[17] S. Manfredi and D. Angeli. Necessary and sufficient conditions for consensus in nonlinear monotone networks with unilateral interactions. Automatica J. IFAC, 77:51-60, 2017. 
[18] R. Goebel and R.G. Sanfelice. Pointwise asymptotic stability in a hybrid system and well-posed behavior beyond Zeno. SIAM J. Control Optim., 56(2):1358-1385, 2018.

[19] M. Jafarian and C. De Persis. Exact formation control with very coarse information. In Proc. 2013 American Control Conference, 2013.

[20] J.P. Hespanha, D. Liberzon, D. Angeli, and E.D. Sontag. Nonlinear normobservability notions and stability of switched systems. IEEE Trans. Automat. Contr., 50(2):154-168, 2005.

[21] D. Liberzon, 2018. private communication.

[22] R.T. Rockafellar and R. J-B Wets. Variational Analysis. Springer, 1998.

[23] H. Brézis. Monotonicity methods in Hilbert spaces and some applications to nonlinear partial differential equations. pages 101-156, 1971.

[24] J.-P. Aubin and A. Cellina. Differential Inclusions. Springer-Verlag, 1984.

[25] C. Henry. An existence theorem for a class of differential equations with multivalued right-hand side. J. Math. Anal. Appl., 41:179-186, 1973.

[26] B. Brogliato, A. Daniilidis, C. Lemaréchal, and V. Acary. On the equivalence between complementarity systems, projected systems and differential inclusions. Systems Control Lett., 55(1):45-51, 2006.

[27] R.E. Bruck Jr. Asymptotic convergence of nonlinear contraction semigroups in Hilbert space. J. Funct. Anal., 18:15-26, 1975. 